\title{
Dispersion Properties of Photonic Crystal Fiber with Four cusped Hypocycloidal Air Holes in Cladding
}

\author{
M.P.Srinivasa Rao ${ }^{1}$, P.Easwaraji ${ }^{2}$ \\ ${ }^{1,2}$ Department of Basic Science and Humanities, GMR Institute of Technology, Rajam, A.P.,India.
}

\begin{abstract}
In this paper, dispersion characteristics of Photonic crystal fiber with four cusped hypocycloidal airhole in cladding (FCH-PCF) are investigated by using fully vectorial effective index method. Computed results show that the dispersion dependence on geometric parameters such as the hole pitch and size parameter. We find the flattened dispersion and zero dispersion wavelengths.
\end{abstract}

Keywords: Dispersion, FCH Photinic crystal fiber, fundamental space filling mode, fully Vectorial effective index method

\section{Introduction}

Dielectric materials with periodic refractive index pattern have the ability to control the propagation properties of electromagnetic waves. Such dielectric materials are known as Photonic crystals or Photonic band gap materials [1]. In recent years, applications of photonic crystals are attracting much attention because of their remarkable and unusual properties [2]. One of the important applications of photonic crystals is the design of novel waveguides known as Photonic crystal fibers (PCF) or Holey fibers or Micro structured fibers [3-4]. PCFs are single material optical fibers with an array of periodic air holes across the cross section running down its entire length by leaving a single lattice site without an air hole, a localized region of higher refractive index is formed. This localized region acts as a waveguide core in which light can be trapped along the axis of the fiber. The presence of the air holes reduces the average refractive index of cladding which leads to conventional guidance similar to communication grade optical fibers. The regularity of photonic crystal cladding allows one to treat as an effective index medium which facilitates the analysis of the properties of the medium and hence the waveguide characteristics. Some of the existing characteristics that have been revolutionized the light guiding feature of PCF are endlessly single mode operation [5], high effective nonlinearity [8], high birefringence [6-7], manageable dispersion [9-10]. Various complicated designs such as different core geometries [11] and multiple air hole diameter in different rings [12] have been studied to achieve ultra flattened dispersion values over wider wavelength bandwidth.

The remarkable properties of PCF arise from the strong wavelength dependence of the effective cladding index [10]. The effective cladding index depends on the geometry of the PCF structure, such as the dimension, air hole shape and pitch. There are many researchers have been reported with different structures such as circular [5,9], elliptical air holes [13], rhombic holes, double air hole unit, four air hole unit so on [1416]. These studies show that the required properties of the PCF can be achieved just by choosing the suitable air hole geometry and pitch.

In this paper, we propose a novel PCF, where the fiber with solid silica core and a cladding with four cusped hypocycloidal (FCH-PCF) air holes along the fiber length. The analysis of the FCH-PCF has been made by using the fully vectorial effective index method. The parameters such as effective index of the cladding and dispersion have been studied for various designs of FCH-PCFs. As result, we report how effective index of the cladding and dispersion of the FCH-PCF depends on its structure. Therefore, we expect that this study will be helpful in the design of photonic crystal fiber with flattened dispersion over wide range of wavelengths and zero dispersion wavelengths.

\section{Fully Vectorial Effective Index Method}

Photonic crystals with periodicity in transverse direction support mode propagation along the longitudinal direction. These modes are referred as space filling modes, because they are infinitely extended in transverse direction. The effective index method is based on the calculation of the effective refractive index of the photonic crystal cladding. The photonic crystal cladding refractive index equals to the modal index of fundamental space filling mode (FSFM).

The effective cladding index is calculated by approximating the square unit cell as an equivalent circular unit cell of outer radius $b$ and inner radius $a$ by keeping constant air filling fraction as shown in Fig. 1 . In the circular unit cell $b=\frac{\Lambda}{\sqrt{\pi}}$ (air filling fraction of square is equal to circle of radius $b$ ) and $a=\frac{d}{2} \sqrt{\frac{3}{8}}$ (area of 
hypocycloid is equal to area of circle radius $a$ ). The characteristic equation for the dispersion relation to find effective cladding index is obtained by solving Maxwell's equations.

$\left(\frac{P_{1}^{\prime}(U)}{U P_{1}(U)}+\frac{I_{1}^{\prime}(W)}{W I_{1}(W)}\right)\left(\frac{n_{s}^{2} P_{1}^{\prime}(U)}{U P_{1}(U)}+\frac{n_{a}^{2} I_{1}^{\prime}(W)}{W I_{1}(W)}\right)=\left[\frac{1}{U^{2}}+\frac{1}{W^{2}}\right]^{2}\left(\frac{\beta}{k}\right)^{2}$

Where $P_{1}=J_{1}(U) Y_{1}(u)-Y_{1}(U) J_{1}(u)$, the parameters $U, W$ and $u$ are defined as $U=k_{o} a \sqrt{n_{s}^{2}-n_{c l}^{2}}$, $W=k_{o} a \sqrt{n_{c l}^{2}-n_{a}^{2}}$ and $u=k_{o} b \sqrt{n_{s}^{2}-n_{c l}^{2}} . n_{s}$ the refractive index of the silica, $n_{a}$ the refractive index of the air, $\beta$ the propagation constant, $k_{o}$ the wave vector in free space, $n_{c l}=n_{e f f} . J, Y$ and $I$ are Bessel functions and the prime indicates differentiation with respect to the argument. The effective index of the FSFM is calculated by solving the scalar wave equation (1) within a unit cell of the FCH-Photonic crystal fiber, centered on one of the hypocycloid air holes as shown in Fig. 1.

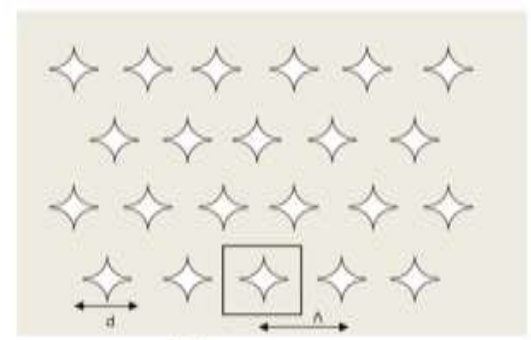

(a)

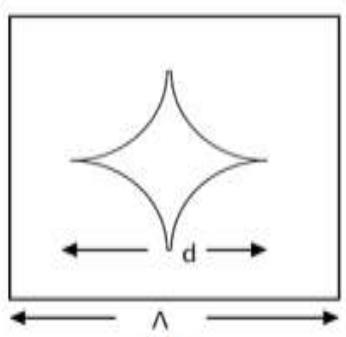

(b)

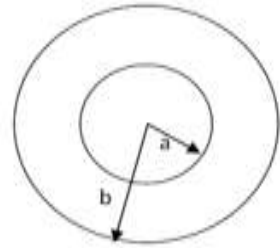

(c)

Figure.1 (a)Two dimensional photonic crystal with air hole size $d$ and pitch $\Lambda$. (b) Square unit cell and (c) Approximation of unit cell into circle.

Therefore, the modal index of FSFM is obtained by solving equation (1) and hence effective cladding index $n_{c l}$ is determined.

\section{Equivalent Step Index Fiber For FCH-PCF}

In order to analyze the propagation characteristics of PCF, it is converted into equivalent step index optical fiber. The refractive index of FSFM is used as the cladding index and known value of the core index $n_{s}$ (silica) are used to refractive indices of the equivalent step index fiber. The propagation constant or modal index of guided wave of FCH Photonic crystal fiber is obtained similar to step index fiber having core index $n_{s}$, cladding index $n_{c l}$ with core size parameter $\rho=0.64 \Lambda$ [10].

The characteristic equation for guided mode in FCH Photonic crystal fiber obtained is similar to the characteristic equation of step index fiber.

$\left(\frac{J_{1}^{\prime}\left(U_{\text {eff }}\right)}{U_{\text {eff }} J_{1}\left(U_{\text {eff }}\right)}+\frac{K_{1}^{\prime}(W)}{W_{\text {eff }} K_{1}(W)}\right)\left(\frac{n_{s}^{2} J_{1}^{\prime}\left(U_{e f f}\right)}{U_{e f f} J_{1}\left(U_{e f f}\right)}+\frac{n_{c l}^{2} K_{1}^{\prime}(W)}{W_{e f f} K_{1}(W)}\right)=\left[\frac{1}{U_{e f f}^{2}}+\frac{1}{W_{e f f}^{2}}\right]^{2}\left(\frac{\beta}{k}\right)^{2}$

And the waveguide parameters are

$U_{e f f}=k_{o} \rho \sqrt{n_{s}^{2}-n_{e f f}^{2}}$ and $W_{e f f}=k_{o} \rho \sqrt{n_{e f f}^{2}-n_{c l}^{2}}$ Where $n_{e f f}$ is the refractive index of the guided mode.

\section{The Effective Refractive Index of the Cladding}

The solution of Eigen value equation (1) gives modal index of FSFM i.e. effective cladding index $n_{c l}$ at different wavelengths. The variation of the effective cladding index as a function of wavelength for different air

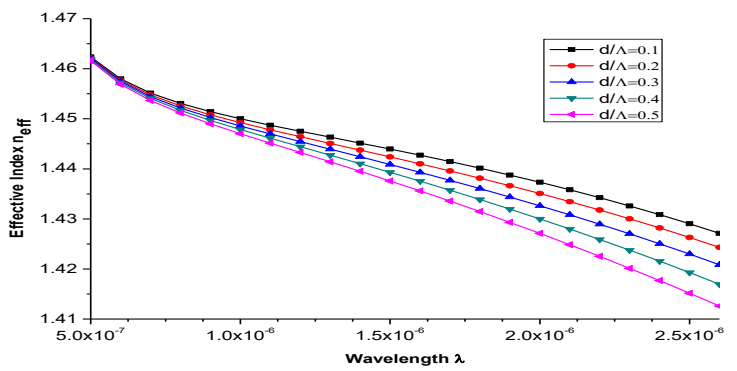

Figure.2. Variation of effective cladding index as a function of wavelength for different $\mathrm{d} / \Lambda$ ration, $\Lambda=4 \mu$ 
hole sizes is shown in Fig.2. It is clear from the figure that effective cladding index decreases with increase air hole size. It is also observed from the Fig. 2.that the modal index of FSFM decreases as the wavelength increases.

\section{Dispersion Calculation}

Chromatic dispersion is a variation in the refractive index of the core with a variation in the wavelength. This variation in refractive index causes the pulses of a modulated light source to broaden when traveling through the fiber. This broadens of pulse nature produces increase in bit error rate and effects quality and speed of the signals. Thus, chromatic dispersion is a major limiting factor in high speed transmission.

We have calculated the Chromatic dispersion $\left(D_{m}\right)$ of the FCH-Photonic crystal fiber through

$$
D_{m}=-\frac{\lambda}{\mathrm{c}} \frac{\mathrm{d}^{2} \mathrm{n}_{\mathrm{eff}}}{\mathrm{d} \lambda^{2}}
$$

Here $\mathrm{n}_{\text {eff }}$ is the refractive index of the guided mode obtained from the equation (2), C is the velocity of light in vacuum.

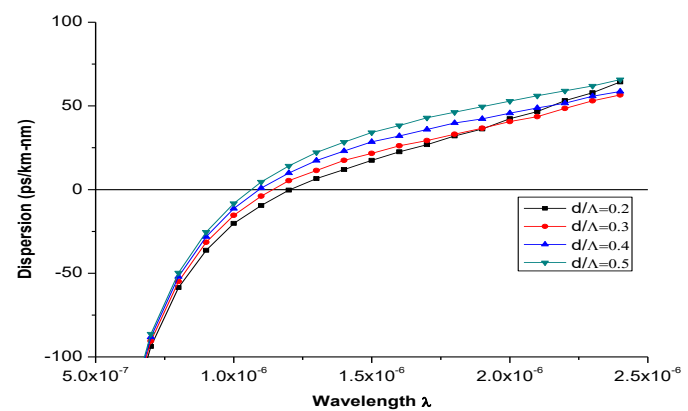

Figure 3 Dispersion as function of wavelength for different relative air-hole sizes $(\mathrm{d} / \Lambda)$ with $\Lambda=4 \mu \mathrm{m}$.

The dispersion plots of FCH-PCF are shown in Fig.3. The Fig.3 shows that the dispersion property is varying between positive and negative values with zero dispersion wavelength. The pitch is taken $4 \mu \mathrm{m}$ and $d / \Lambda$ ratio $0.2,0.3,0.4$ and 0.5 are considered. From the Fig 3 we could observe that four zero dispersion wavelengths. It is also observed that as $d / \Lambda$ increases, zero dispersion wavelength shifts towards lower wavelength. These dispersion characteristics are summarized in Table 1. The amplitude of dispersion variation lies approximately between $-100 \mathrm{ps} / \mathrm{nm} / \mathrm{km}$ and $60 \mathrm{ps} / \mathrm{nm} / \mathrm{km}$ within the wavelength range from $0.5 \mu \mathrm{m}$ to $2.4 \mu \mathrm{m}$. So, it is possible to shift the zero dispersion wavelength by change the geometrical parameters such as $\Lambda$ and $d$.

Table 1: Zero dispersion wavelength of FCH-PCF with $\Lambda=4 \mu \mathrm{m}$

\begin{tabular}{|l|l|}
\hline \multicolumn{1}{|c|}{$/ \boldsymbol{\Lambda}$} & $\begin{array}{l}\text { Zero dispersion wavelength } \\
(\mathbf{Z D W})\end{array}$ \\
\hline 0.2 & $1.21 \mu \mathrm{m}$ \\
\hline 0.3 & $1.15 \mu \mathrm{m}$ \\
\hline 0.4 & $1.09 \mu \mathrm{m}$ \\
\hline 0.5 & $1.06 \mu \mathrm{m}$ \\
\hline
\end{tabular}

The waveguide dispersion $\left(\mathrm{D}_{\mathrm{w}}\right)$ is defined as [10]

$D_{W}=-\frac{n_{e f f} \Delta_{e f f}}{0.0003}\left(V_{e f f} \frac{d^{2}\left(V_{e f f} b_{e f f}\right)}{d V_{e f f}^{2}}\right) \mathrm{ps} / \mathrm{km}-\mathrm{nm}$

Where $\frac{d^{2}\left(V_{\text {eff }} b_{\text {eff }}\right)}{d V^{2}}=\frac{2 U^{2} \mathcal{K}_{1}}{V^{2} W^{2}}\left\{\left[3 W^{2}-2 \mathcal{K}_{1}\left(W^{2}-U^{2}\right)\right]+W\left(W^{2}+\mathcal{K}_{1} U^{2}\right)\left(\mathcal{K}_{1}-1\right) \frac{K_{0}(W)+K_{1}(W)}{K_{1}(W)}\right\}$

And $\mathcal{K}_{1}=\frac{K_{1}^{2}(w)}{K_{0}(W) K_{1}(W)}$

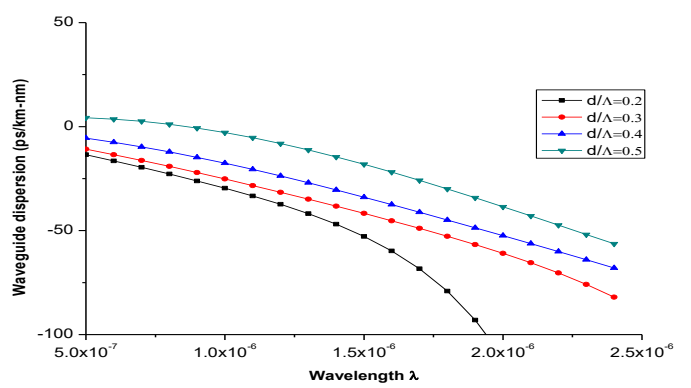


Figure 4 Waveguide dispersion as function of wavelength for different relative air-hole sizes $(\mathrm{d} / \Lambda)$ with $\Lambda=4 \mu \mathrm{m}$ The variation of waveguide dispersion is shown in Fig. 4 for different $d / \Lambda$ ratios.

It is observed from the Fig. 4 that the negative dispersion increases with decrease in $d / \Lambda$ ratio. The waveguide dispersion is negative for longer wavelengths.

The total dispersion in Photonic crystal fibers is due to the waveguide dispersion and material dispersion. Thus the total dispersion $D=D_{m}+D_{w}$. The total dispersion is well represented in Fig.5.

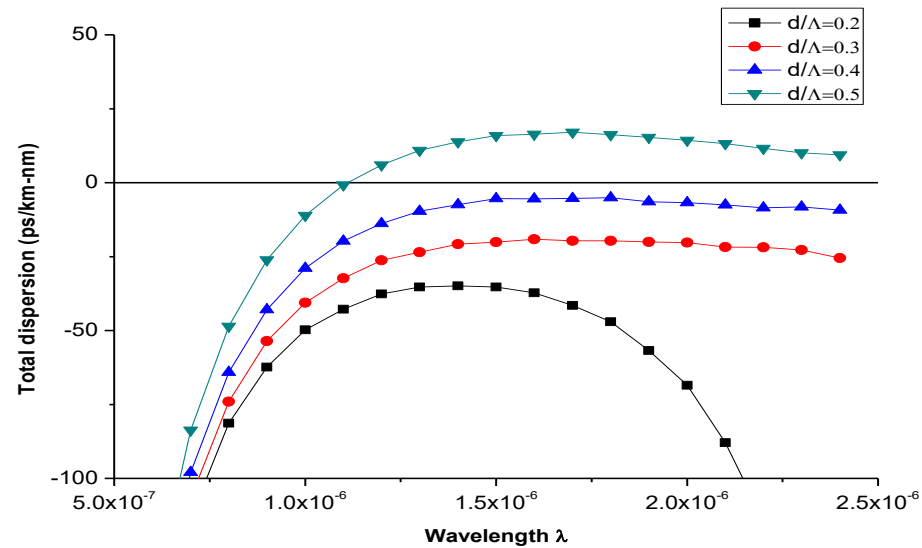

Figure 5 Total Dispersion as function of wavelength for different relative air-hole sizes $(\mathrm{d} / \Lambda)$ with $\Lambda=4 \mu \mathrm{m}$

From Fig.5 it is obvious to understand that one can design dispersion flattened PCF over a broad range of wavelengths simply by tuning the cladding parameters $d$ and $\Lambda$. We have obtained the flattened positive dispersion response above the wavelength $1.15 \mu \mathrm{m}$ for $d / \Lambda$ ratio 0.5 . Further it is also observed that flattened negative dispersion above the wavelength $1.15 \mu \mathrm{m}$ for $d / \Lambda$ ratio 0.4 and 0.3 . This dispersion-flattened response of PCF can be utilized in dense-Wavelength Division Multiplexing (DWDM) based optical communication systems.

Large negative dispersion in Photonic crystal fibers can be obtained not only at a single wavelength, but also for a wide range of wavelengths. A large index contrast between core and cladding permits a high dispersion over a broad wavelength range. In the case of Photonic crystal fibers, a large index contrast is easily obtained, as it depends on the cladding parameters air hole size $d$ and air hole spacing $\Lambda$. The interesting fact that can be observed from the Fig. 5 is large negative dispersion at shorter and longer wavelengths and flattened negative dispersion at intermediate wavelengths for $d / \Lambda=0.2$. These large values of negative dispersion could be used to compensate the anomalous dispersion of conventional single mode fiber. This means that Photonic crystal fiber can compensate the dispersion caused by standard single-mode fiber. Thus, large negative dispersion of Photonic crystal fiber makes them useful candidates for use as dispersion compensating fibers in optical communication links. These types of fibers opened up new area in nonlinear optics such as ultra-broad supercontinuum generation in silica micro structured fibers. This type of low dispersion property is highly preferred for the few cycle pulse generation.

\section{Conclution}

In this study, we report the analysis of FCH-PCF emphasizing its dispersion properties. The Fully vectorial effective index method is used to obtain the effective refractive index of $2 \mathrm{D}$ photonic crystal cladding. The Eigen value equation is used to analyze the FCH PCF. It is shown that flattened dispersion with zero wavelength dispersion can be achieved by choosing the values of $d / \Lambda$ in the cladding. Flattened dispersion from FCH PCFs will promote their application in DWDM communication systems, and the high negative chromatic dispersion in FCH PCF will prove useful for dispersion compensating devices.

\section{References}

[1] E. Yablonovitch, Photonic band gap structures, J. Opt. Soc. Am. B., 10, 1993, 283-295.

[2] Ekmel Ozbay, Irfan Bulu, Koray Aydin, Humeya Calgayan, Kean Guven, Physics and Applications of Photonic Crystals, Photonics and Nanostructures Fundamentals and Applications, 2, 2004, 87-95.

[3] J. Broeng, D. Mogilevstev, S. E. Barkou and A. Bjakle, Photonic Crystal Fibers: a new class of optical waveguides, Opt. Fiber Tech., 5, 1999, 305-330.

[4] P. St. J. Russel, Photonic-Crystal Fibers, J of Lightwave Tech., 24, 2004, 4729-4749.

[5] T. A. Birks, J. C. Knight, and P. St. J. Russell, Endlessly single-mode photonic crystal fiber, Opt. let., 22, $1997,961-963$.

[6] J. H. Liou, S. S. Huang, and C. P. Yu Loss-reduced highly birefringent selectively liquid-filled photonic crystal fibers, Opt. Comm. 283, 2010, 971-974. 
[7] T. P. Hansen, J. Broeng, S. E. B. Libori, E. Enudsen, A. Bjarklev, J. R. Jensen and H. Simpson, Highly Birefringent Index-Guiding Photonic Crystal Fibers, IEEE Photon. Technl. Lett.,13, 2001, 588-590.

[8] John M. Dudley and J. Roy Taylor, Ten years of nonlinear optics in photonic crystal fibre, Nature Photonics, 3, 2009. 85-90.

[9] R.K.Sinha and Anshu D. Varshney, Dispersion properties of Photonic crystal fiber : Comparison by scalar and fully vectorial effective index methods, Optical and Quantum Electronics, .37, 2005,711-722.

[10] Shailendra K, Varshney, M.P.Singh and R.K.Singha, Propagation characteristics of Photonic crystal fiber, J. of Opt. Commun. 24, 2003, 856-858.

[11] K. Saitoh, N. J. Florous, and M. Koshiba, Theoretical realization of holey fiber with flat chromatic dispersion and large mode area: an intriguing defected approach, Opt. Lett., 31, 2006,26-28.

[12] T.-L. Wu and C.-H. Chao, A Novel Ultraflattened Dispersion Photonic Crystal Fiber, IEEE Photon. Technol. Lett.,17, $2005,67-69$.

[13] M. J. Steel and R. M. Osgood Jr., Polarization and dispersive properties of elliptical-hole photonic crystal fibers, J. Lightwave Technol. 19, 2001, 495-503.

[14] B. Hu, M. Lu, W. Li, K. Zou, Z. Zhou, A. Lin, N. Li, High birefringent rhombic-hole photonic crystal fibers, Appl. Opt., 49, 2010, 6098-6100.

[15] D. Chen, G. Wu, Highly birefringent photonic crystal fiber based on a double-hole unit, Appl. Opt., 49, 2010, 1682-1690.

[16] Aihan Yin, Yisheng Ding, Lei Xiong, Design and analysis of a photonic crystal fiber with four-hole unit, Optik, 125, 2014.70687071..

[17] M. P. Srinivasa Rao, B.Prasad, P. khastiger and S.P.Ojha, Modal cutoff condition for a optical wavegude with a hypocycloidal cross section, Microwave and optical technology let., .14, 199, 177-180. 